MICROSCOPY

\section{Easing access to the nanoscale}

Cox et al. report a new high-resolution microscopy method for living cells that should be more readily accessible to cell biologists than existing super-resolution microscopy approaches.

Super-resolution microscopy

techniques offer the promise of imaging cell biological processes with increased resolution, but a limiting factor has been the ability to acquire images quickly enough to follow dynamic processes in live cells. Moreover, current methods either use specialized microscopes or require the use of wavelengths and intensities that damage live samples, thus limiting their utility in vivo.

The method described by Cox et al. involves performing a Bayesian statistical analysis of images that are generated by high-speed imaging of fluorescent proteins using a standard wide-field microscope with xenon arc lamp illumination. The authors analysed data that included overlapping fluorophores using a range of effects, including blinking and bleaching events, as well as fluorophores being added or removed in the cell. This analysis method allowed them to perform localization microscopy in living cells with a spatial resolution of $50 \mathrm{~nm}$ and a temporal resolution of $4 \mathrm{~s}$.

To test the method, the authors imaged podosomes in a human cell line expressing mCherry-tagged truncated talin. Podosomes are cytoskeletal structures involved in cell migration and adhesion and are associated with the degradation of the extracellular matrix. They consist of an actin filament core surrounded by a ring of integrinassociated proteins, such as talin and vinculin. Interestingly, Cox et al. show, using this approach, that podosomes often have a polygonal structure, that they form smaller ring-type structures and that they are highly
$3 \mathrm{~B}$ analysis image of a truncated talin construct in podosomes. This image is generated from 200 frames ( $4 \mathrm{~s}$ ) of raw data. Scale bar is $2 \mu \mathrm{m}$. Image courtesy of S. Cox, King's College London, UK.

this

methodology should readily increase the resolution that is possible with live-cell imaging

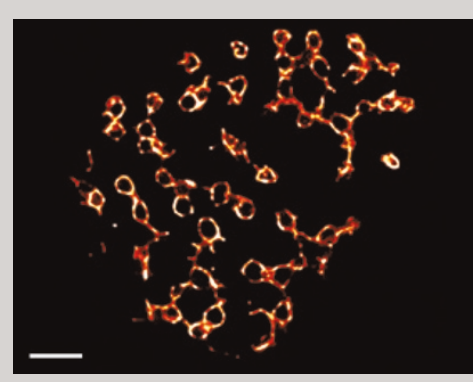

dynamic, dissociating and reforming over a timescale of tens of seconds. These characteristics were previously unappreciated, as the integrin-associated ring was thought to be round, and podosomes were thought to dissociate over several minutes.

Thus, this methodology should readily increase the resolution that is possible with live-cell imaging experiments using fluorescent proteins and reveal previously unappreciated levels of complexity in biological systems.

\section{Kim Baumann}

ORIGINAL RESEARCH PAPER Cox, S. et al.

Bayesian localization microscopy reveals

nanoscale podosome dynamics. Nature Methods 4 Dec 2011 (doi:10.1038/nmeth.1812) 